\title{
Application of Compressive Sensing to Asymptotic Waveform Evaluation for Fast Frequency-Sweep Analysis of Electromagnetic Scattering Problems
}

\author{
Meng Kong, ${ }^{1,2}$ Ming-Sheng Chen $\mathbb{D}^{1,},{ }^{1,2}$ Xin-Yuan Cao, ${ }^{1,2}$ and Xian-Liang Wu ${ }^{1,2}$ \\ ${ }^{1}$ Anhui Province Key Laboratory of Simulation and Design for Electronic Information System, Hefei Normal University, Hefei, China \\ ${ }^{2}$ School of Electronic Information and Electrical Engineering, Hefei Normal University, Hefei, China \\ Correspondence should be addressed to Ming-Sheng Chen; chenms@ustc.edu.cn
}

Received 11 December 2018; Accepted 10 April 2019; Published 22 April 2019

Academic Editor: N. Nasimuddin

Copyright (C) 2019 Meng Kong et al. This is an open access article distributed under the Creative Commons Attribution License, which permits unrestricted use, distribution, and reproduction in any medium, provided the original work is properly cited.

To reduce the computing resource of full-scale impedance matrix and its high-order derivatives in traditional Asymptotic Waveform Evaluation (AWE), compressive sensing (CS) is applied to AWE for fast and accurate frequency-sweep analysis of electromagnetic scattering problems. In CS framework, some prior knowledge is extracted by constructing and solving undetermined equation of 0 -order surface induced current, so that coefficients about high-order induced current can be accurately obtained by the prior knowledge, and finally the wide-band radar cross section (RCS) is calculated. Numerical results of two-dimensional objects and bodies of revolution (BOR) were presented to the show the efficiency of the proposed method.

\section{Introduction}

During the past decades, Asymptotic Waveform Evaluation (AWE) has always been a prevalent frequency domain technique for solving electromagnetic scattering problems over a wide frequency band. Under the framework of moment of method (MOM), AWE was applied to fast calculate the wideband radar cross section (RCS) of conducting and dielectric objects [1-4]. The fast multipole method (FMM) and multilevel fast multipole method (MLFMM) in conjunction with AWE has been successfully used for handling the electromagnetic scattering problems of electrically large objects $[5,6]$. In AWE, the induced current is transformed into Taylor series around a given frequency, and the Padé approximation is used to expand the radius of convergence; unfortunately, the solution is much memory consuming and time consuming since the high derivatives of the dense impedance matrix must be stored. To improve the computing performance of the traditional AWE, a sparse preprocessing technique based on lifting wavelet transform (LWT) [7] was applied to AWE. However, the full-scale dense impedance matrix must be filled before sparse transform, which is the major time consuming operation.

Recently, compressive sensing (CS) $[8,9]$ has been successfully introduced to MOM for accelerating analysis of electromagnetic scattering problems over a wide incident angle [10]. In [11, 12], based on CS, a small scale impedance matrix is obtained to serve as the measurement matrix in CS; thus an undetermined equation was constructed to solve EM problems efficiently.

In this paper, CS theory is used in conjugation with AWE (AWE-CS) to calculate wide-band RCS of two-dimensional perfectly electric conduct (PEC) objects and bodies of revolution (BOR), by which we proposed a new scheme to fast construct and solve an undetermined equation over a wide frequency band. In this new scheme, some undetermined equations are constructed firstly by fast filling small scale impedance matrix and its high-order derivatives. Secondly, with the prior knowledge proposed by calculating the real induced currents, the coefficients of high-order current can be accurately reconstructed with the help of suitable sparse transform and recovery algorithm in CS. Finally, RCS of 
objects is obtained effectively at any frequency point within the given frequency band.

\section{Theory and Formulation}

2.1. Asymptotic Waveform Evaluation. For PEC objects illuminated by an incident electric field $\left(E^{\text {inc }}\right)$, the electric field integral equation (EFIE) can be shown as

$$
\widehat{\mathbf{n}} \times \mathbf{E}^{\text {inc }}=-\widehat{\mathbf{n}} \times \eta L(\mathbf{J})
$$

where $\widehat{\mathbf{n}}$ is the outward normal of the PEC object surface, $\eta$ denotes the free-space wave impedance, and $\mathbf{J}$ is the surface current density. The integral operator $\boldsymbol{L}$ is given by

$$
L(\mathbf{J})=-j k \iint_{s}\left\{\mathbf{J}+\frac{1}{k^{2}} \nabla\left(\nabla^{\prime} \cdot \mathbf{J}\right)\right\} G d S^{\prime}
$$

in which $k$ and $G$ are wave number and Green's function in free-space, respectively.

MOM is used for discrete equation (1), which can be reduced to a matrix equation

$$
[\mathbf{Z}(k)]_{N \times N}[\mathbf{I}(k)]_{N \times 1}=[\mathbf{V}(k)]_{N \times 1}
$$

where $[\mathbf{V}]_{N \times 1}$ is the excitation vector including $N$ segments, $[\mathbf{I}]_{N \times 1}$ denotes currents vector which is of the same size as excitation vector, and $\mathbf{Z}$ is $N \times N$ impedance matrix.

For body of revolution (BOR), BOR-MOM can transform (1) into two scalar linear integral equations in two tangential directions along the generator curve of BOR [13], respectively, and the matrix form can be given by

$$
[\mathbf{Z}(k)]_{2 N \times 2 N}[\mathbf{I}(k)]_{2 N \times 1}=[\mathbf{V}(k)]_{2 N \times 1}
$$

By AWE, the solution of (3) or (4) over wide-band can be obtained by a few operation steps as follows. $\mathbf{I}(k)$ is expanded around $k_{0}$ (an interesting frequency point) into a Taylor series:

$$
\mathbf{I}(k)=\sum_{i=0}^{P} \mathbf{m}_{i}\left(k-k_{0}\right)^{i}
$$

where $\mathbf{m}_{i}=\mathbf{I}^{(i)}\left(k_{0}\right) / i !(\boldsymbol{i}=0,1,2, \cdots P)$ can be solved by

$$
\begin{aligned}
& {\left[\mathbf{Z}\left(k_{0}\right)\right]\left[\mathbf{m}_{\mathbf{0}}\right]=\left[\mathbf{V}\left(k_{0}\right)\right]} \\
& {\left[\mathbf{Z}\left(k_{0}\right)\right]\left[\mathbf{m}_{q}\right]=\left[\frac{\mathbf{V}^{(q)}\left(k_{0}\right)}{q !}-\sum_{i=1}^{q} \frac{\mathbf{Z}^{(i)}\left(k_{0}\right) \mathbf{m}_{q-i}}{i !}\right]} \\
& (q=1,2, \cdots P)
\end{aligned}
$$

To expand convergence radius of the Taylor series, $\mathbf{I}(k)$ is approximated with a rational Padé function

$$
\mathbf{I}(k)=\frac{\sum_{i=0}^{L} \mathbf{a}_{i}\left(k-k_{0}\right)^{i}}{\sum_{j=0}^{R} \mathbf{b}_{j}\left(k-k_{0}\right)^{j}}
$$

where $L+R=P, \mathbf{a}_{i}$ and $\mathbf{b}_{j}$ are unknown coefficients, and they can be solved by

$$
\left[\begin{array}{cccc}
\mathbf{m}_{L} & \mathbf{m}_{L-1} & \cdots & \mathbf{m}_{L-R+1} \\
\mathbf{m}_{L+1} & \mathbf{m}_{L} & \cdots & \mathbf{m}_{L-R+2} \\
\mathbf{m}_{L+2} & \mathbf{m}_{L+1} & \cdots & \mathbf{m}_{L-R+3} \\
\vdots & \vdots & \ddots & \vdots \\
\mathbf{m}_{L+R-1} & \mathbf{m}_{L+R-2} & \cdots & \mathbf{m}_{L}
\end{array}\right]\left[\begin{array}{c}
\mathbf{b}_{1} \\
\mathbf{b}_{2} \\
\mathbf{b}_{3} \\
\vdots \\
\mathbf{b}_{R}
\end{array}\right]=-\left[\begin{array}{c}
\mathbf{m}_{L+1} \\
\mathbf{m}_{L+2} \\
\mathbf{m}_{L+3} \\
\vdots \\
\mathbf{m}_{L+R}
\end{array}\right]
$$

$$
\left[\begin{array}{c}
\mathbf{a}_{0} \\
\mathbf{a}_{1} \\
\mathbf{a}_{2} \\
\vdots \\
\mathbf{a}_{L}
\end{array}\right]=\left[\begin{array}{ccccc}
\mathbf{m}_{0} & \mathbf{0} & \mathbf{0} & \cdots & \mathbf{0} \\
\mathbf{m}_{1} & \mathbf{m}_{0} & \mathbf{0} & \cdots & \mathbf{0} \\
\mathbf{m}_{2} & \mathbf{m}_{1} & \mathbf{m}_{0} & \cdots & \mathbf{0} \\
\vdots & \vdots & \vdots & \ddots & \vdots \\
\mathbf{m}_{L} & \mathbf{m}_{L-1} & \mathbf{m}_{L-2} & \cdots & \mathbf{m}_{L-R}
\end{array}\right]\left[\begin{array}{c}
1 \\
\mathbf{b}_{1} \\
\mathbf{b}_{2} \\
\vdots \\
\mathbf{b}_{R}
\end{array}\right]
$$

Substituting $\mathbf{a}_{i}$ and $\mathbf{b}_{j}$ into (8), the discrete current can be obtained over a wide frequency band.

2.2. CS Theory. In the framework of CS theory, a sparse signal can be compressed to measurements, and the length of the measurements is much smaller than the nominal number of discrete points. Finally, the original signal can be recovered by solving an optimization problem.

Consider a nonsparse signal $[\mathbf{X}]_{N \times 1}$, which can be transformed to a sparse signal $[\boldsymbol{\alpha}]_{N \times 1}$ in $[\Psi]_{N \times N}$

$$
[\boldsymbol{\alpha}]_{N \times 1}=[\Psi]_{N \times N}[\mathbf{X}]_{N \times 1}
$$

where $[\Psi]_{N \times N}$ is a transformed matrix, and $[\mathbf{X}]_{N \times 1}$ can be named $K$-sparse signal if $[\boldsymbol{\alpha}]_{\mathrm{N} \times 1}$ contain $K$ nonzero elements.

As a compressed representation of the original signal, $[\mathbf{Y}]_{M \times 1}$ can be obtained by

$$
\begin{array}{r}
{[\mathbf{Y}]_{M \times 1}=[\boldsymbol{\Phi}]_{M \times N}[\boldsymbol{\alpha}]_{N \times 1}=[\boldsymbol{\Phi}]_{M \times N}[\boldsymbol{\Psi}]_{N \times N}[\mathbf{X}]_{N \times 1}} \\
(M \ll N)
\end{array}
$$

where $[\boldsymbol{\Phi}]_{M \times N}$ is a measurement matrix, and $[\mathbf{Y}]_{M \times 1}$ is the measurement result.

To accurately reconstruct $[\mathbf{X}]_{N \times 1}$, the number of measurements $M$ must satisfy $M \approx K \ln (N / K) \ll N$, and $[\Phi]_{M \times N}$ is required to meet restricted isometry property (RIP). In the step of reconstruction, undetermined equation (12) can obtain unique result by solving optimization problems as follows:

$$
\begin{array}{ll}
\min & \|\Psi \mathbf{X}\|_{1} \\
\text { s.t. } & \boldsymbol{\Phi} \Psi \mathbf{X}=\mathbf{Y}
\end{array}
$$

2.3. The New Method. As we know, the traditional AWE technique must operate some computationally expensive steps, which contain the filling and storage of dense impedance matrix and its high derivatives. Inspired by CS, a fast new scheme is constructed based MOM to improve the efficiency of traditional AWE. Unlike the solution of $\mathbf{m}_{\mathbf{0}}$ in (6) using 
MOM, the new method constructs an undetermined equation:

$$
\left[\mathbf{Z}\left(k_{0}\right)\right]_{M \times N}\left[\mathbf{m}_{\mathbf{0}}\right]_{N \times 1}=\left[\mathbf{V}\left(k_{0}\right)\right]_{M \times 1} \quad(M \ll N)
$$

in which $\left[\mathbf{Z}\left(k_{0}\right)\right]_{M \times N}$ is a small scale impedance matrix that can be used to represent the interaction obtained between the random $M$ weight functions and the whole $N$ basic functions; $\left[\mathbf{V}\left(k_{0}\right)\right]_{M \times 1}$ represent the corresponding excitations on $M$ field points.

For nonsparse induced current $\left[\mathbf{m}_{0}\right]_{N \times 1}$, an orthogonal matrix $[\Psi]_{N \times N}$ can be applied as

$$
\begin{aligned}
{[\boldsymbol{\Psi}]_{N \times N}\left[\mathbf{m}_{\mathbf{0}}\right]_{N \times 1} } & =[\boldsymbol{\alpha}]_{N \times 1} \\
{\left[\mathbf{m}_{\mathbf{0}}\right]_{N \times 1} } & =\left[\boldsymbol{\Psi}^{\mathrm{T}}\right]_{N \times N}[\boldsymbol{\alpha}]_{N \times 1}
\end{aligned}
$$

Substituting (15) and (16) into (14),

$$
\begin{aligned}
& {\left[\mathbf{Z}^{c s}\left(k_{0}\right)\right]_{M \times N}[\boldsymbol{\alpha}]_{N \times 1}} \\
& \quad=\left[\mathbf{Z}^{c s}\left(k_{0}\right)\right]_{M \times N}[\boldsymbol{\Psi}]_{N \times N}\left[\mathbf{m}_{\mathbf{0}}\right]_{N \times 1}=\left[\mathbf{V}\left(k_{0}\right)\right]_{M \times 1}
\end{aligned}
$$

where $\left[\mathbf{Z}^{c s}\left(k_{0}\right)\right]_{M \times N}=\left[\mathbf{Z}\left(k_{0}\right)\right]_{M \times N}\left[\Psi^{\mathrm{T}}\right]_{N \times N}$.

Equation (17) can be explained under the CS framework as follows: $\left[\mathbf{Z}^{c s}\left(k_{0}\right)\right]_{M \times N}$ is a measurement matrix that satisfies the RIP since the Toeplitz property of impedance matrix $[14]$, and $\left[\mathbf{V}\left(k_{0}\right)\right]_{M \times 1}$ is the known measurement of the sparse signal $[\boldsymbol{\alpha}]_{N \times 1}$. To reconstruct the unknown $[\boldsymbol{\alpha}]_{N \times 1},(13)$ can be solved by the orthogonal matching pursuit (OMP) technique [15], and finally $\left[\mathbf{m}_{\mathbf{0}}\right]_{N \times 1}$ can be obtained by (16).

Due to the similarities between (6) and (7), the high-order coefficients $\left[\mathbf{m}_{q}\right]_{N \times 1}(q=1,2, \ldots, P)$ can be obtained using the method described above for $P$ times.

In traditional AWE, the computational complexity for solving $\left[\mathbf{m}_{i}\right]_{N \times 1}(\mathrm{i}=0,1,2,3, \ldots, P) P+1$ times by iteration method is $O\left((P+1) S N^{2}\right)$, where $S$ is the number of iteration steps, and the complexity of impedance matrix filling is $O\left(N^{2}\right)$. However, the filling complexity of proposed method only needs $O(M N)$, and the reconstructed complexity of $\left[\mathbf{m}_{i}\right]_{N \times 1}$ $(\mathrm{i}=0,1,2,3, \ldots, P)$ by OMP is $O((P+1) K M N)$, where $K<<$ $S, M<<$. Therefore, the complexity of the new method will be far less than the traditional AWE.

\section{Result and Discussion}

To validate the effectiveness of the proposed method, we analyze scattering characteristics of various objects on the personal computer (Intel core i7-5500U@2.40GHz, RAM $8.0 \mathrm{GHz}$ ). The error described by relative root-mean-square error (R-RMSE) is defined as

$$
\operatorname{R-RMSE}(i)=\frac{\left\|\widetilde{\mathbf{m}}_{i}-\mathbf{m}_{i}\right\|_{2}}{\left\|\mathbf{m}_{i}\right\|_{2}} \times 100 \% \frac{}{(i=0,1,2, \ldots, P)}
$$

in which $\widetilde{\mathbf{m}}_{i}$ is $i$-order reconstruct coefficient by OMP, and $\mathbf{m}_{i}$ is real $i$-order coefficient by traditional MOM. In the following examples, the results over a wide-band are solved by AWE with Padé approximation $(L=4, R=3)$.

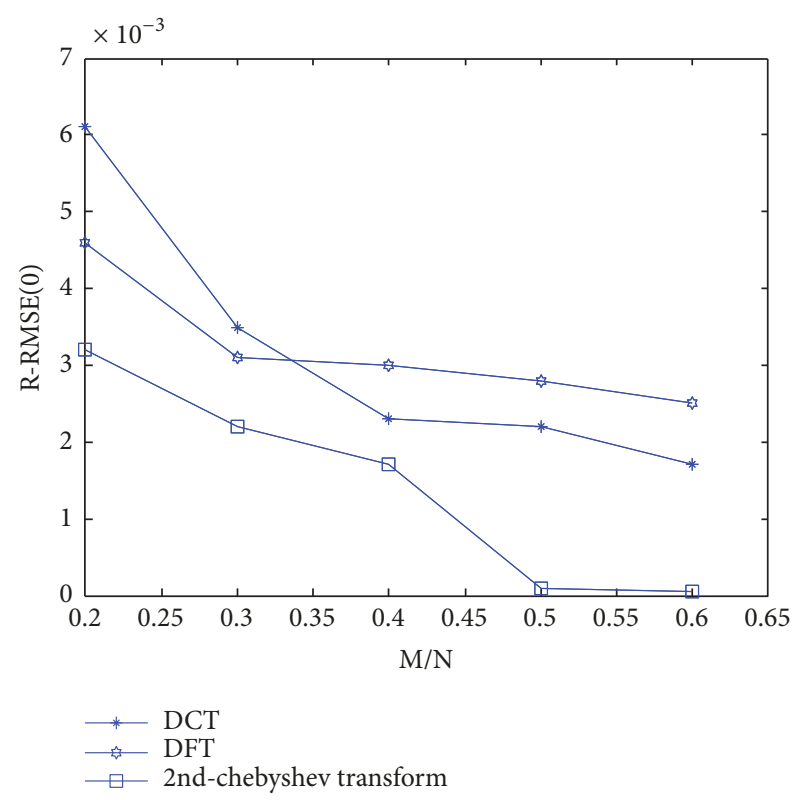

FIGURE 1: Relationship between $\mathrm{M} / \mathrm{N}$ and the reconstructed error in different sparse transform matrices.

As the first example, a perfect electrical conducting cylinder with a radius of $0.5 \mathrm{~cm}$ is considered, which is illuminated by a TM plane wave, and the perimeter of cylinder is divided into 200 segments $(N=200)$. For the proposed method, $\widetilde{\mathbf{m}}_{\mathbf{0}}$ is first solved to determine key prior knowledge at $30.0 \mathrm{GHz}$. In Figure 1 , as $M / N$ increases, the $\mathrm{R}-\mathrm{RMSE}(0)$ reduces in different sparse transform matrices of the proposed method, which contain Discrete Cosine Transform (DCT), Discrete Fourier Transform (DFT), and 2nd-Chebyshev Transform. To balance the R-RMSE(0) and the computational complexity, $M / N=0.3(\mathrm{M}=60)$ and 2 ndChebyshev Transform are selected to solve $\widetilde{\mathbf{m}}_{\mathbf{0}}$ by CS method, which means the fill of matrix in (14) dropped to $30 \%$ of a dense impedance matrix in (3). Figure 2 shows the induced current obtained by CS method in comparison with that of MOM.

Based on the proposed method, the coefficients $\left[\mathbf{m}_{q}\right]_{N \times 1}$ $(q=1,2, \ldots, 7)$ can also be solved by CS method, in which the key prior knowledge $(M / N=0.3,2$ nd-Chebyshev Transform is selected as the sparse transform matrix) was obtained from the first step. As shown in Figure 3, the R-RMSE of all $i$-order coefficients are less than $2 \%$.

As shown in Figure 4, with the help of solved coefficients $\mathbf{m}_{i}$, the so called AWE-CS and AWE are used to analyze RCS frequency response of the cylinder with $0.03 \mathrm{GHz}$ increments from 28.5 to $31.5 \mathrm{GHz}$, and the result agrees well with that of the direct solution of MOM at each of the frequency points, in which the frequency step is $0.3 \mathrm{GHz}$. On the CPU time aspects, the direct solution of MOM, AWE, and AWE-CS consumed 55.16s, 16.05s, and 7.74s, respectively.

Based on CS, the choice of sparse transform plays an important role in accuracy of the reconstructed solution $[16,17]$. For different signals, some empirical knowledge has been obtained in selecting suitable sparse transform 


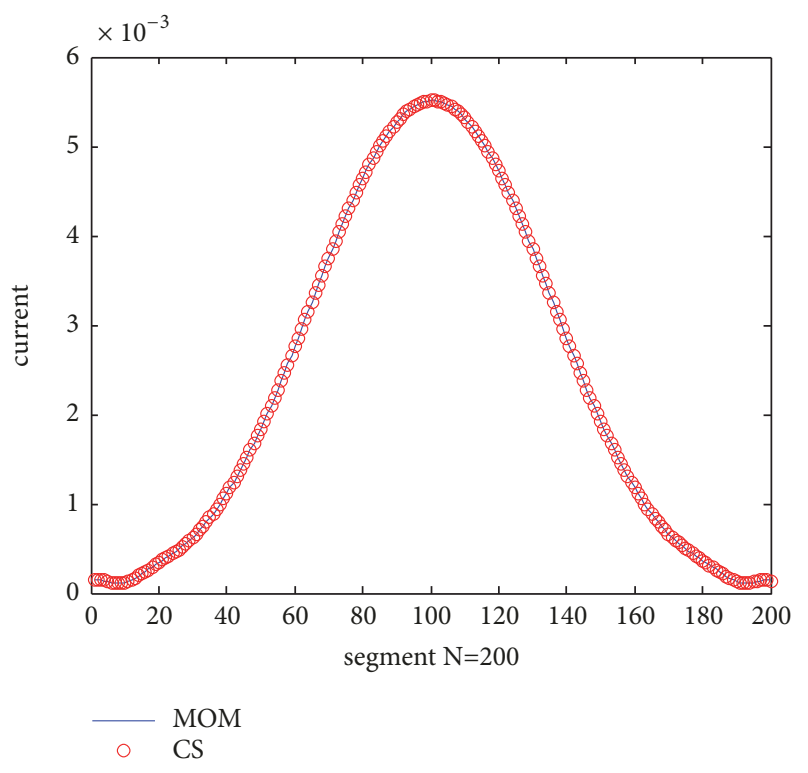

Figure 2: Current distribution $\left(\mathbf{m}_{\mathbf{0}}\right)$ of the PEC cylinder at different segments.

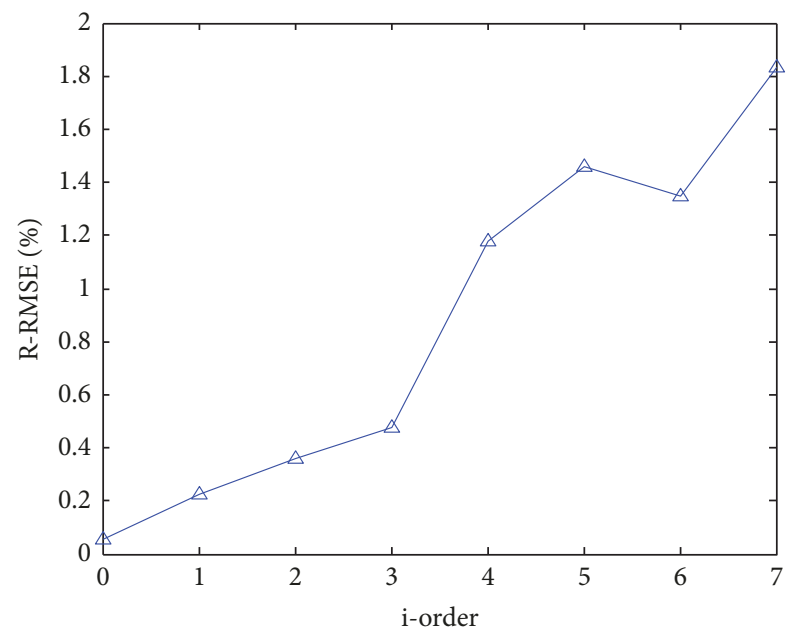

FIGURE 3: The R-RMSE of coefficients $\mathbf{m}_{i}(\mathrm{i}=0,1,2, \ldots, 7)$ between MOM and CS method.

matrices; for example, DCT and 2nd-Chebyshev Transform are suitable for slowly changing signal, and DFT is suitable for signals containing rich frequency components, and DWT shows advantage in abrupt changes signal [18], etc. In the first example, the key prior knowledge is from the solution procedure of the cylinder induced current; however, the parameters can be seen as reference value for other objects, so that the general picture of unknown induced current can be obtained to provide basis for setting optimizing parameters to the proposed method.

Considering a PEC square cylinder with a side length of $1 \mathrm{~cm}$ illuminated by a TM plane wave as the second example, the perimeter of the objects is divided into 256 equally spaced segments $(N=256)$. As the test of the proposed method, $\mathrm{M} / \mathrm{N}=30 \%$ and 2nd-Chebyshev Transform are determined

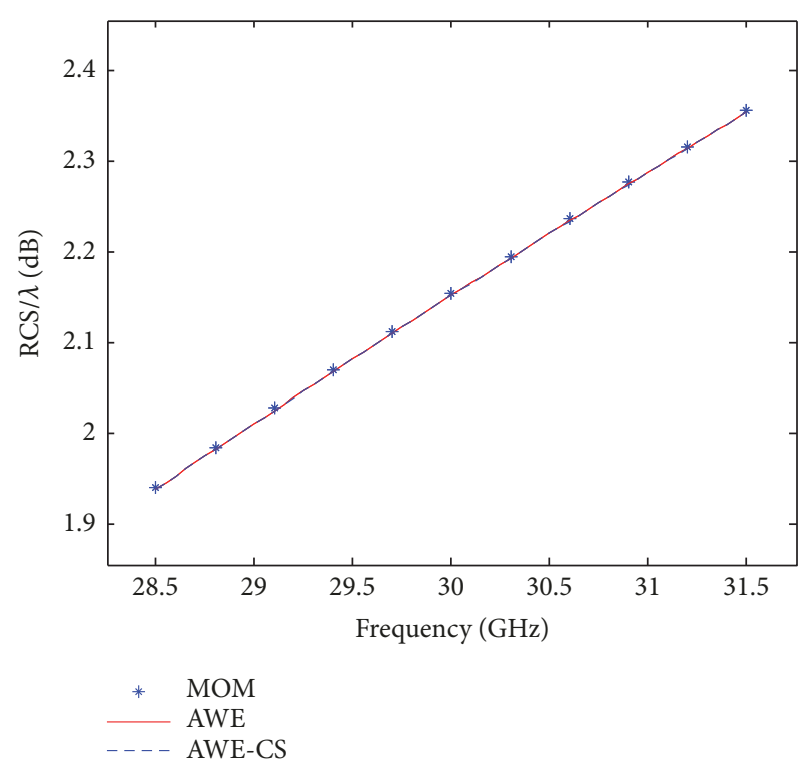

FIGURE 4: RCS frequency response of PEC cylinder with radius of $0.5 \mathrm{~cm}$.

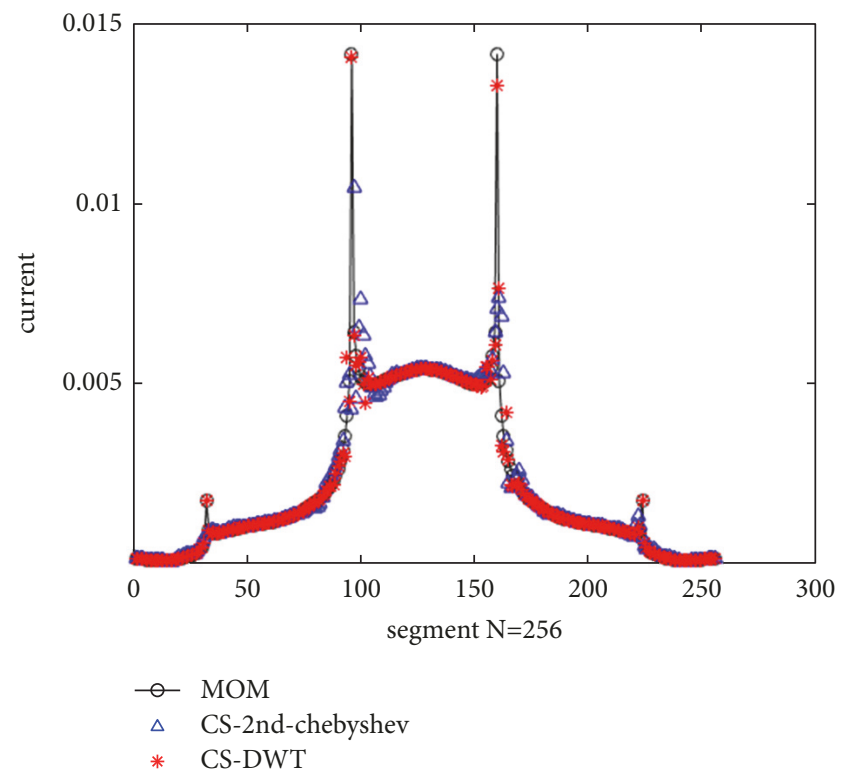

FIGURE 5: Current distribution $\left(\mathbf{m}_{\mathbf{0}}\right)$ of the PEC square cylinder at different segments (CS-2nd-Chebyshev: $M / N=30 \%$, RRMSE=6.73\%; CS-DWT: $M / N=30 \%$, R-RMSE $=0.58 \%$ ).

for solving current distribution $\left(\mathbf{m}_{\mathbf{0}}\right)$ at $30 \mathrm{GHz}$. In Figure 5, we can see the general picture of test result is abrupt changes signal, so DWT is selected as the sparse transform matrix to obtain the result by CS. As shown in Figure 5, the result of the proposed method agrees well with the result of MOM. To obtain the RCS frequency response from 27 to $33 \mathrm{GHz}$, the direct method consumed 150.37s with a frequency step of $0.5 \mathrm{GHz}$, whereas AWE and AWE-CS consumed 44.23 and 19.14 s with a frequency step of $0.06 \mathrm{GHz}$, respectively, and the results are shown in Figure 6. 


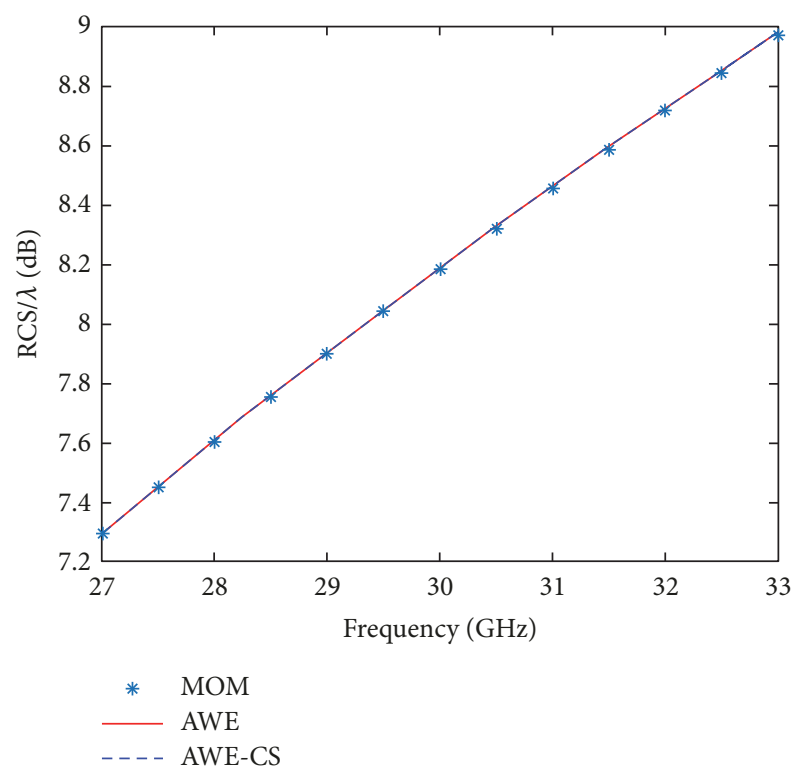

FigURE 6: RCS frequency response of PEC square cylinder from 27 to $33 \mathrm{GHz}$.

The following two examples are presented to demonstrate the performance of AWE-CS for BOR. In the examples, $M / N=30 \%$ is set and 2nd-Chebyshev Transform is chosen to support the proposed method. Firstly, a $10 \mathrm{~cm}$ long PEC almond is considered, which was illuminated by an axially incident plane wave with vertical polarization, and the generator of PEC almond is divided into 200 segments $(N=200)$. As shown in Figure 7, the direct method (BORMOM) consumed 1770.32s to obtain the solution from 0.4 to $0.8 \mathrm{GHz}$ with a frequency step of $20 \mathrm{MHz}$. With one expansion point at $0.6 \mathrm{GHz}$, the AWE and AWE-CS give efficient solutions with $4 \mathrm{MHz}$ increments over the entire band in 387.66s and 147.31s, respectively.

Finally, a PEC missile-like object with a length of $1 \mathrm{~cm}$ is considered, which was illuminated by an axially incident plane wave with vertical polarization, and the generator is divided into 150 segments $(\mathrm{N}=150)$. In Figure 8, results of three methods (BOR-MOM, AWE, and AWE-CS) agree well with each other as RCS frequency response is calculated from 2.0 to $4.0 \mathrm{GHz}$. With a frequency step of $0.1 \mathrm{GHz}, \mathrm{BOR}-\mathrm{MOM}$ takes 1572.69 s to obtain the solution. With a expansion point at $3.0 \mathrm{GHz}$, AWE and AWE-CS produce accurate solutions with $20 \mathrm{MHz}$ increments over the entire band in 328.91s and 138.89 s, respectively.

\section{Conclusion}

Based on CS, a new novel scheme is proposed for fast analysis of RCS broadband response. The proposed method constructs a small scale impedance matrix and its highorder derivatives instead of filling the full dense matrix in traditional AWE, and the extract key prior knowledge in initial step is used for accurate reconstruction of highorder coefficients. The simulations from two-dimensional

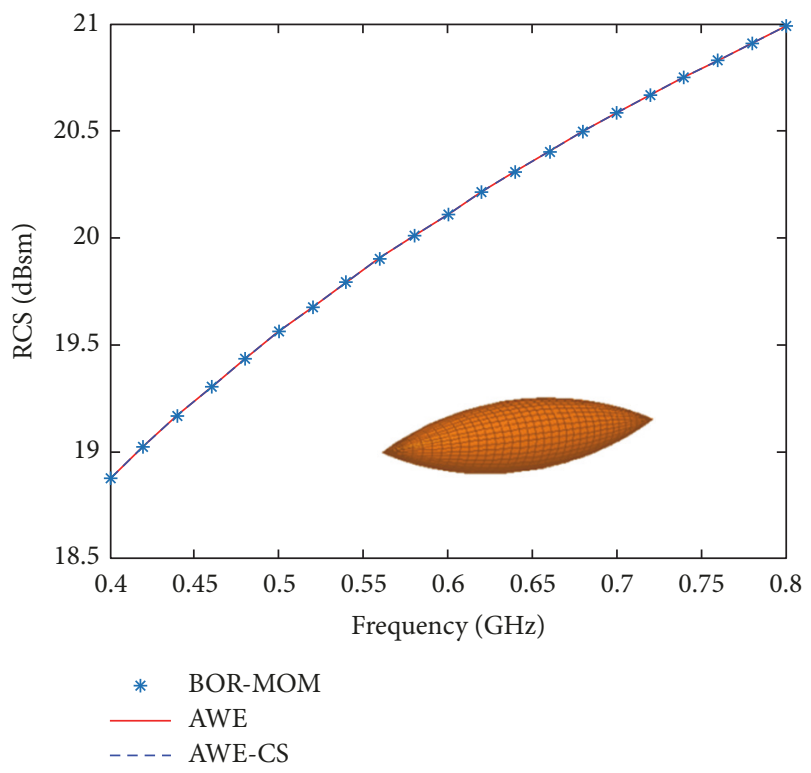

FIGURE 7: RCS frequency response of the PEC almond from 0.4 to $0.8 \mathrm{GHz}$.

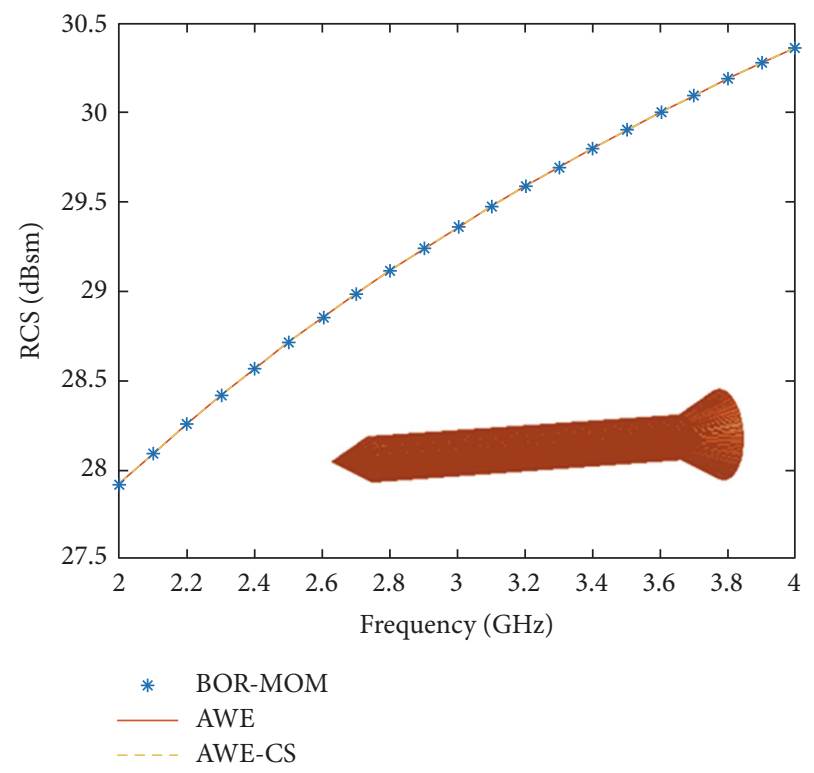

FIGURE 8: RCS frequency response of the PEC missile-like object from 2.0 to $4.0 \mathrm{GHz}$.

and BOR PEC objects have demonstrated the high efficiency and accuracy of the proposed method.

\section{Data Availability}

The data used to support the findings of this study are available from the corresponding author upon request.

\section{Conflicts of Interest}

The authors declare that there are no conflicts of interest regarding the publication of this paper. 


\section{Acknowledgments}

This work was supported by the National Natural Science Foundation of China under Grant Nos. 61801163 and 61701163, Anhui Provincial Natural Science Foundation under Grant No. 1808085QF204, Anhui Science and Technology Project under Grant No. 201904a05020061, and the Key Project of Provincial Natural Science Research of University of Anhui Province of China under Grant KJ2018A0489.

\section{References}

[1] C. J. Reddy, M. D. Deshpande, C. R. Cockrell, and F. B. Beck, "Fast RCS computation over a frequency band using method of moments in conjunction with asymptotic waveform evaluation technique," IEEE Transactions on Antennas and Propagation, vol. 46, no. 8, pp. 1229-1233, 1998.

[2] D. Jiao, X.-Y. Zhu, and J.-M. Jin, "Fast and accurate frequencysweep calculations using asymptotic waveform evaluation and the combined-field integral equation," Radio Science, vol. 34, no. 5, pp. 1055-1063, 1999.

[3] Y. B. Wu, J. X. Zhang, and Q. X. Sheng, "Frequency sweep computation of half-space scattering using asymptotic waveform evaluation," Microwave and Optical Technology Letters, vol. 58, no. 3, pp. 495-498, 2016.

[4] R. Y. Jeong, P. I. Hong, K. W. Lee et al., "Fast frequency sweep using asymptotic waveform evaluation technique and thin dielectric sheet approximation," IEEE Transactions on Antennas Propagation, vol. 64, no. 5, pp. 1800-1806, 2016.

[5] X. C. Wei, Y. J. Zhang, and E. P. Li, "The hybridization of fast multipole method with asymptotic waveform evaluation for the fast monostatic RCS computation," IEEE Transactions on Antennas and Propagation, vol. 52, no. 2, pp. 605-607, 2004.

[6] B.-Y. Wu and X.-Q. Sheng, "Application of asymptotic waveform evaluation to hybrid FE-BI-MLFMA for fast RCS computation over a frequency band," IEEE Transactions on Antennas \& Propagation, vol. 61, no. 5, pp. 2597-2604, 2013.

[7] C. Ming-Sheng, W. Xian-liang, and S. Wei, "Asymptotic waveform evaluation technique based on fast lifting wavelet transform," Applied Computational Electromagnetics Society Journal, vol. 21, no. 1, pp. 99-104, 2006.

[8] D. L. Donoho, “Compressed sensing," IEEE Transactions on Information Theory, vol. 52, no. 4, pp. 1289-1306, 2006.

[9] Y. Tsaig and D. L. Donoho, "Extensions of compressed sensing," Signal Processing, vol. 86, no. 3, pp. 549-571, 2006.

[10] M. S. Chen, F. L. Liu, H. M. Du, and X. L. Wu, "Compressive sensing for fast analysis of wide-angle monostatic scattering problems," IEEE Antennas and Wireless Propagation Letters, vol. 10, no. 3, pp. 1243-1246, 2014.

[11] Z. Wang, B. Wang, and M. Tan, "Use of Compressed Sensing in analysis of electric field integral equation by the Method of Moments," in Proceedings of the 2014 IEEE International Symposium on Antennas and Propagation \& USNC/URSI National Radio Science Meeting, pp. 2000-2001, Memphis, Tenn, USA, July 2014.

[12] M. Kong, M. Sheng Chen, B. Wu, and X. L. Wu, "Fast and stabilized algorithm for analyzing electromagnetic scattering problems of bodies of revolution by compressive sensing," IEEE Antennas and Wireless Propagation Letters, vol. 16, pp. 198-201, 2017.

[13] M. Kong, M. Chen, L. Zhang, X. Cao, and X. Wu, "Efficient solution to electromagnetic scattering problems of bodies of revolution by compressive sensing," Chinese Physics Letters, vol. 33, no. 1, pp. 136-139, 2016.

[14] W. U. Bajwa, J. D. Haupt, G. M. Raz, S. J. Wright, and R. D. Nowak, "Toeplitz -structured compressed sensing matrices," in Proceedings of the IEEE/SP Workshop on Statistical Signal Processing IEEE, 2007.

[15] J. F. Theis, A. Jung, and G. C. Puntonet, "Signal recovery from partial information via orthogonal matching pursuit," IEEE Transactions on Information Theory, vol. 15, no. 2, pp. 419-439, 2007.

[16] X. Y. Cao, M. S. Chen, and X. L. Wu, "Sparse transform matrices and their application in the calculation of electromagnetic scattering problems," Chinese Physics Letters, vol. 30, no. 2, Article ID 028401, 2013.

[17] H.-M. Du, M.-S. Chen, and X.-L. Wu, "The structure of the sparse basis function in compressive sensing for fast analysis of wide-angle monostatic scattering problems," in Proceedings of the 2012 International Conference on Microwave and Millimeter Wave Technology, ICMMT 2012, pp. 910-912, China, May 2012.

[18] Z. Wang and B. Z. Wang, "Application of compressed sensing theory in the method of moments," Acta Physica Sinica, vol. 22, no. 12, Article ID 120202, pp. 202-207, 2014. 


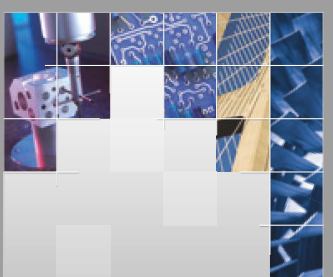

\section{Enfincering}
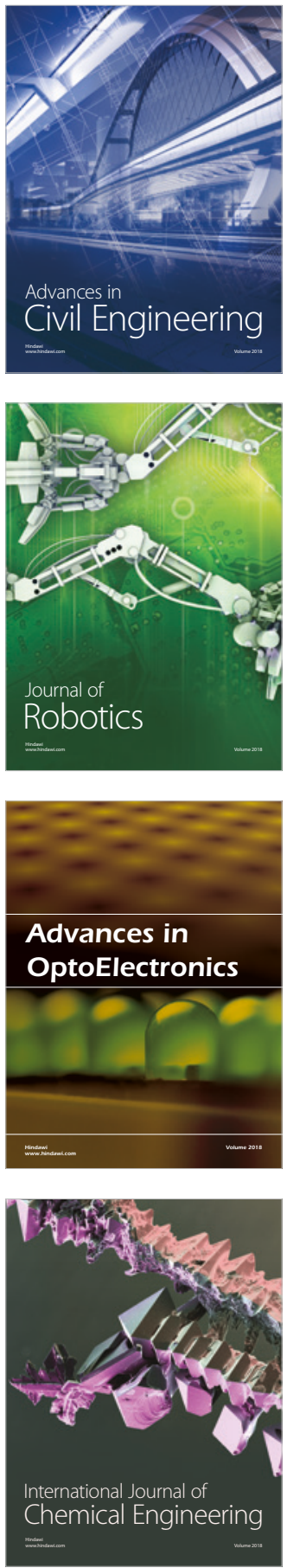

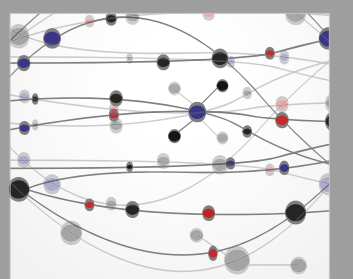

\section{Rotating \\ Machinery}

The Scientific World Journal

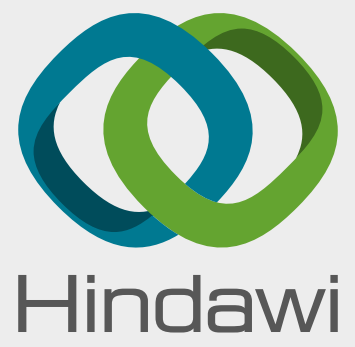

Submit your manuscripts at

www.hindawi.com
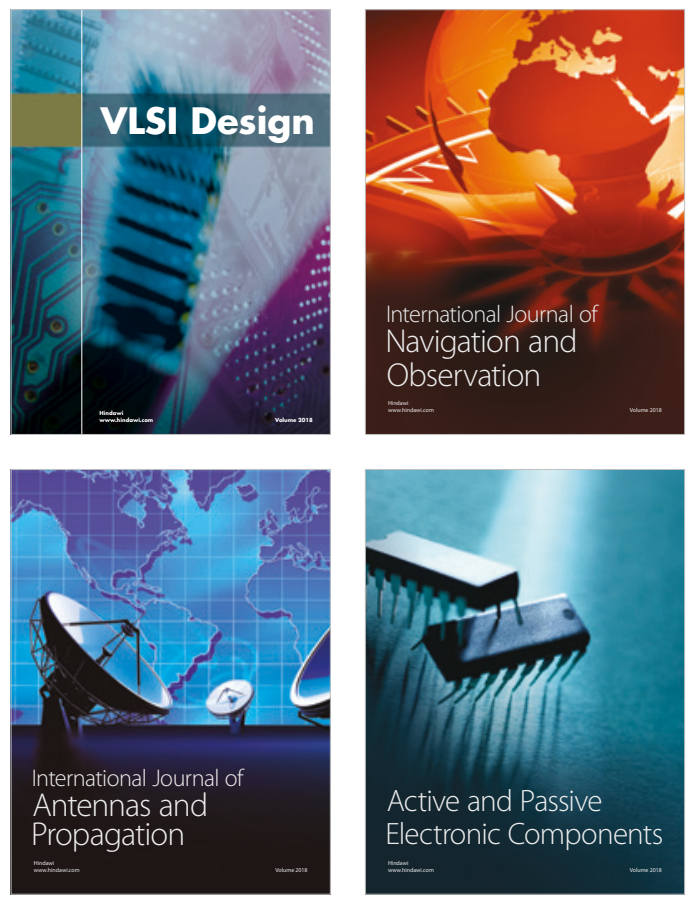
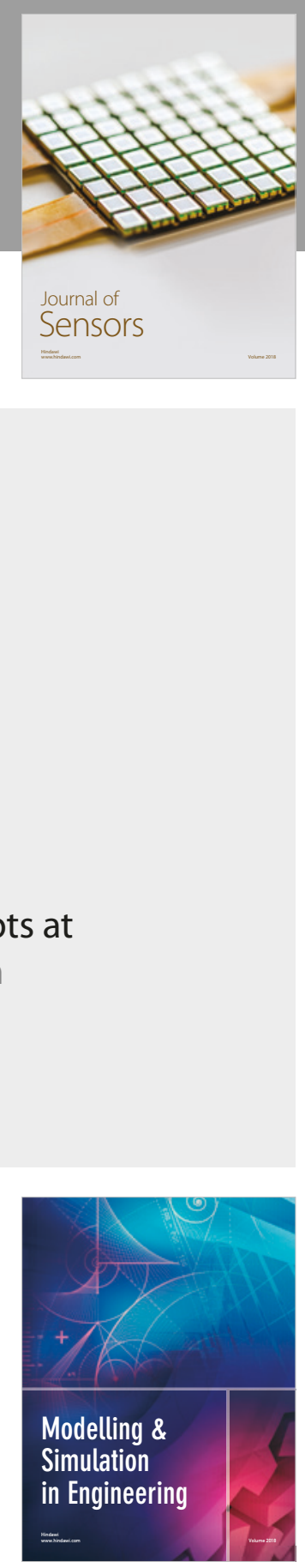

\section{Advances \\ Multimedia}
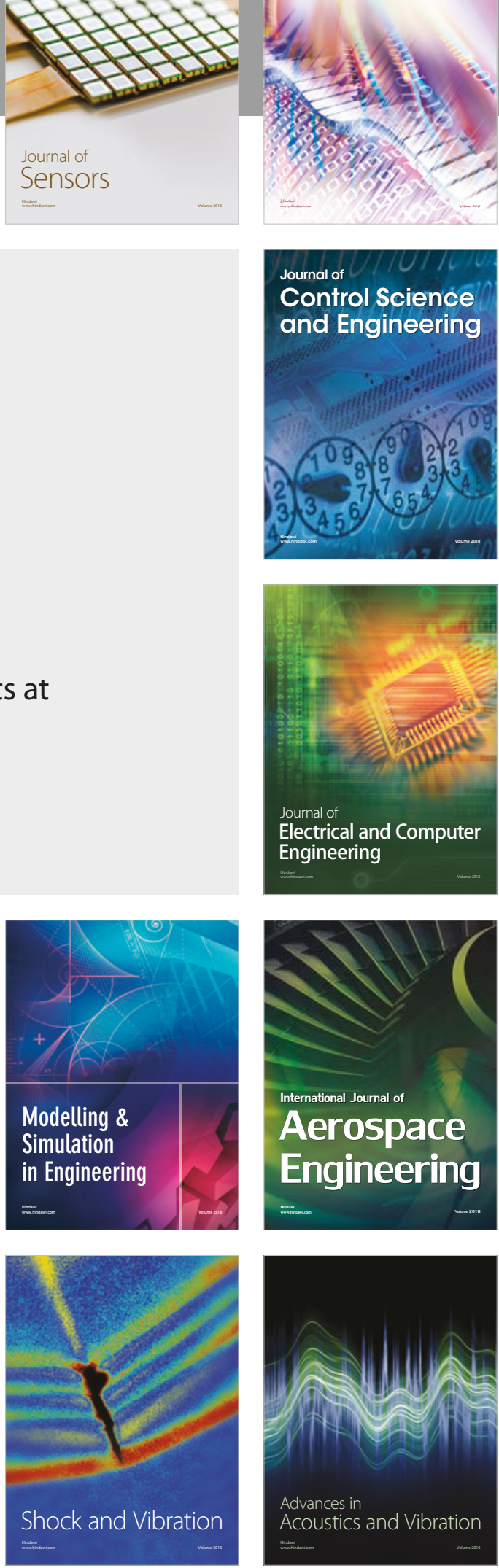